RUB-TPII-03/2015

\title{
Matching lightcone- and anomaly-sum-rule predictions for the pion-photon transition form factor
}

\author{
A. G. Oganesian,,${ }^{1,2}$ A. V. Pimikov,${ }^{1,3}$ N. G. Stefanis,${ }^{4}$ O. V. Teryaev,${ }^{1 *}$ \\ 1 Bogoliubov Laboratory of Theoretical Physics, \\ Joint Institute for Nuclear Research, 141980, Dubna, Russia, \\ ${ }^{2}$ Institute of Theoretical and Experimental Physics, 117218, Moscow, Russia \\ ${ }^{3}$ Institute of Modern Physics, Chinese Academy of Sciences, Lanzhou, 730000, China \\ ${ }^{4}$ Institut für Theoretische Physik II, Ruhr-Universität Bochum, D-44780 Bochum, Germany
}

\begin{abstract}
The pion-photon transition form factor is studied by employing two types of Sum Rules: Light Cone Sum Rules (LCSR) and Anomaly Sum Rules (ASR). By comparing the predictions for the pion-photon transition form factor, obtained from these two approaches, the applicability limit of the LCSRs at low momenta is determined. Reciprocally, the ASR threshold dependence on the momentum was extracted using our LCSR-based method in combination with two different types of pion distribution amplitudes and found that at higher $Q^{2}$ it approaches a constant.
\end{abstract}

*Electronic addresses: armen@itep.ru, pimikov@theor.jinr.ru, stefanis@tp2.ruhr-uni-bochum.de, teryaev@theor.jinr.ru. 


\section{Introduction}

The measurement of the transition form factor (TFF) $F_{\pi \gamma}\left(Q^{2}\right)$ at $4<Q^{2}<40 \mathrm{GeV}^{2}$ by the BaBar Collaboration [1] in 2009 has showed very unexpected results: although at $Q^{2}<10 \mathrm{GeV}^{2}$ the collected data are in agreement with previous experiments, the trend of the measured TFF at $Q^{2} \gtrsim 10 \mathrm{GeV}^{2}$ strongly exceeds the predicted asymptotic limit $[2,3]$. This is given by

$$
F_{\pi \gamma}^{\text {asy }}\left(Q^{2}\right)=\frac{\sqrt{2} f_{\pi}}{Q^{2}}+\mathcal{O}\left(1 / Q^{4}\right)
$$

and deviations from it are challenging the validity of the factorization property of hard exclusive processes within Quantum Chromodynamics (QCD). On the other hand, the more recent data of the Belle Collaboration [4] of the year 2012 do not indicate such a large growth of the scaled TFF at high $Q^{2}$. This significant difference in the data trend, stimulated a number of theoretical investigations of the TFF at various momentum transfers $Q^{2}$ some questioning the validity of the BaBar data, e.g., [5], while other proposals attempting to rationalize this peculiar TFF behavior, for example, $[6,7]$.

Perturbative Quantum Chromodynamics (pQCD) in the leading-order (LO) approximation of the collinear factorization approach to the pion TFF predicts [2,3]:

$Q^{2} F_{\pi \gamma}\left(Q^{2}\right)=\left(\sqrt{2} f_{\pi} / 3\right) \int_{0}^{1} d x \varphi_{\pi}^{(2)}\left(x, Q^{2}\right) / x=\left(\sqrt{2} f_{\pi} / 3\right)\left\langle x^{-1}\right\rangle_{\pi}$, where $\varphi_{\pi}^{(2)}\left(x, \mu^{2}\right)$ is the pion distribution amplitude (DA) of twist two encoding the nonperturbative partonic interactions. This important relation imposes a crucial constraint on the profile of the pion DA in terms of its inverse moment and holds in any theoretical framework based on collinear QCD factorization - see [8] for a comprehensive discussion. However, it is not known at which momentum value, "asymptotia" is effectively reached and the TFF starts to scale with $Q^{2}$.

Expressing the DA in terms of the conformal Gegenbauer expansion,

$$
\varphi_{\pi}^{(2)}\left(x, \mu^{2}\right)=\sum_{n=0,2,4, \ldots}^{\infty} a_{n}\left(\mu^{2}\right) \psi_{n}(x),
$$

where $\psi_{n}(x)=6 x(1-x) C_{n}^{(3 / 2)}(2 x-1)$ with $\varphi_{\pi}^{\text {asy }}(x)=6 x(1-x) \equiv 6 x \bar{x}$ are the eigenfunctions of the Efremov-Radyushkin-Brodsky-Lepage (ERBL) evolution equation [2,9], one can evolve the TFF at any higher scale $Q^{2} \geq \mu^{2}$ by determining the nonperturbative coefficients $a_{n}\left(\mu^{2}\right)$ at the scale of choice. At infinitely large $Q^{2}$, the pion DA assumes its asymptotic form $\varphi_{\pi}^{\text {asy }}(x)$ giving rise to the asymptotic result for the TFF, viz., Eq. (1), in which all possible perturbative and nonperturbative corrections are absent. To account for these corrections at finite $Q^{2}$ values, one has to apply more sophisticated approaches, like lightcone sum rules (LCSR), developed in [10,11] and applied by Bakulev, Mikhailov, Pimikov, and Stefanis (BMPS) in [12-18] (see also [19-21]). 
In an independent parallel development by Klopot, Oganesian, and Teryaev (KOT) [22-25], it was shown that the photon transition form factors of pseudoscalar mesons can be studied by means of anomaly sum rules (ASRs) which are based on the dispersive representation of the axial anomaly. This procedure is closely connected to the treatment of the vector-vector-axial triangle graph amplitude [26-28], in which the axial current is assumed to represent the pion and the two vector currents represent the real and the virtual photon. The key element of the ASR method is that it does not rely upon the factorization hypothesis and, in this sense, it is directly related to the first principles of QCD and the Dynamical Chiral Symmetry Breaking (DCSB) and the concomitant mass generation of hadrons. In addition, KOT have extended the analytic continuation of the ASRs (and herewith the TFF computation) to the timelike region [29]. This is particularly important because the low-momentum timelike regime is unreachable in the conventional pQCD approach.

In this paper we want to compare the two approaches, i.e., ASRs vs. LCSRs, and match their predictions for the pion-photon TFF. The scope of the analysis is to identify the treacherous points of each of these methods and determine their accuracy limits. The paper is organized as follows. In the next two sections, we will briefly recall the basic ingredients of both methods, starting with the LCSRs in Sec. 2 and continuing with the ASRs in Sec. 3. The comparison of the predictions obtained with the two methods will be addressed in Sec. 4, while Sec. 5 is devoted to our conclusions.

\section{LCSR approach}

The behavior of the TFF can be obtained from a detailed formalism (called in the following BMPS for short), developed in a series of papers [12-18,30]. This formalism combines the dispersive approach of LCSRs pioneered in $[10,11]$ (see also $[20,21,31]$ ) with QCD sum rules which employ nonlocal condensates (NLC)s [32-38]. The NLC QCD SRs are used to derive the pion DA, while the LCSRs serve our purpose twofold: first, to take into account the hadronic content of the low-virtuality photon and second, to incorporate contributions from QCD perturbation theory and higher-twist corrections. Within QCD, the pion-photon transition form factor for two off-shell photons $F^{\gamma^{*} \gamma^{*} \pi^{0}}$ is given by the matrix element

$$
\int d^{4} z e^{-i q_{1} \cdot z}\left\langle\pi^{0}(P)\left|T\left(J_{\mu}(z) J_{\nu}(0)\right)\right| 0\right\rangle=i \epsilon_{\mu \nu \alpha \beta} q_{1}^{\alpha} q_{2}^{\beta} F^{\gamma^{*} \gamma^{*} \pi^{0}}\left(Q^{2}, q^{2}\right),
$$

where $J_{\mu}$ is the quark electromagnetic current and both photons are assumed to have finite virtualities $q_{1}^{2}=-Q^{2}>>q_{2}^{2}=q^{2}>0$. The LCSR [10,11] for this matrix element 
reads

$$
\begin{aligned}
Q^{2} F^{\gamma^{*} \gamma^{*} \pi}\left(Q^{2}, q^{2}\right)= & \frac{\sqrt{2}}{3} f_{\pi}\left[\frac{Q^{2}}{m_{\rho}^{2}+q^{2}} \int_{x_{0}}^{1} \exp \left(\frac{m_{\rho}^{2}-Q^{2} \bar{x} / x}{M^{2}}\right) \bar{\rho}\left(Q^{2}, x\right) \frac{d x}{x}\right. \\
& \left.+\int_{0}^{x_{0}} \bar{\rho}\left(Q^{2}, x\right) \frac{Q^{2} d x}{\bar{x} Q^{2}+x q^{2}}\right] .
\end{aligned}
$$

Here, the integration limits are defined by $x_{0}=Q^{2} /\left(Q^{2}+s_{\rho}\right)$ and $s=\bar{x} Q^{2} / x$, where $s_{\rho} \simeq 1.5 \mathrm{GeV}^{2}$ is the effective threshold in the vector channel, and $M^{2}$ is the Borel parameter, with $m_{\rho}=0.77 \mathrm{GeV}$ denoting the physical mass of the $\rho$ meson. The main theoretical ingredient in the above sum rule is the spectral density $\bar{\rho}\left(Q^{2}, x\right)=$ $\left(Q^{2}+s\right) \rho^{\text {pert }}\left(Q^{2}, s\right)$, where

$$
\rho^{\mathrm{pert}}\left(Q^{2}, s\right)=\frac{1}{\pi} \operatorname{Im} F^{\gamma^{*} \gamma^{*} \pi^{0}}\left(Q^{2},-s-i \varepsilon\right)=\rho_{\mathrm{tw}-2}+\rho_{\mathrm{tw}-4}+\rho_{\mathrm{tw}-6}+\ldots
$$

The leading-twist spectral density was studied up to the next-to-next-to-leading order (NNLO) level of pQCD, i.e., $\rho_{\mathrm{tw}-2}=\rho_{\mathrm{LO}}+\rho_{\mathrm{NLO}}+\rho_{\mathrm{NNLO}_{\beta_{0}}}+\cdots$, albeit only the $\beta_{0}$ part of the NNLO term is known [39] (see also [5]). For the next-to-leadingorder (NLO) term $\rho_{\mathrm{NLO}}$, we employ the expression computed in [5] with the correction pointed out in [20], whereas the leading-order contribution is given by the Born term. To compute the spectral density, one takes recourse to the hard-scattering amplitudes, which are calculable within pQCD as a series expansion in terms of the coupling parameter $a_{s}\left(\mu_{\mathrm{R}}^{2}\right)=\alpha_{s}\left(\mu_{\mathrm{R}}^{2}\right) /(4 \pi)$, where $\mu_{\mathrm{R}}^{2}$ is the renormalization scale. In the works of the BMPS team, the renormalization and the factorization scale have been identified and set equal to $Q^{2}$. This choice avoids the appearance of scheme-dependent numerical coefficients which play no important role in our considerations. All said, the leading twist-two expression for the pion-photon TFF has the perturbative expansion

$$
F_{\gamma^{*} \gamma^{*} \pi^{0}}^{\mathrm{tw}-2} \sim\left[T_{\mathrm{LO}}+a_{s}\left(\mu^{2}\right) T_{\mathrm{NLO}}+a_{s}^{2}\left(\mu^{2}\right) T_{\mathrm{NNLO}_{\beta_{0}}}+\ldots\right] \otimes \varphi_{\pi}^{(2)}\left(x, \mu^{2}\right)
$$

where $\otimes \equiv \int_{0}^{1} d x$. The TFF is dominated by the first nontrivial perturbative term proportional to $T_{\mathrm{NLO}}$ and the twist-four contribution. On the other hand, the overall uncertainties have various sources. These are: (i) uncertainties related to the particular pion DA model adopted, (ii) twist-four uncertainties, (iii) estimated uncertainties related to the twist-two contribution at the $\mathrm{NNLO}_{\beta_{0}}$ level, and (iv) uncertainties induced by the twist-six term. The latter two uncertainties are taken into account by means of their sum because for $M^{2} \approx 0.75 \mathrm{GeV}^{2}$ they are comparable in size and have a relatively small magnitude but enter with opposite signs [16]. For larger values of the Borel parameter around $M=1.5 \mathrm{GeV}^{2}$, as used for instance in the LCSR analysis in [20], the uncertainties related to the twist-six term turn out to be small.

For definiteness, we use for the calculation of the TFF the family of the pion DAs determined in [30] with the help of QCD sum rules with NLCs and the nonlocality 
parameter $\lambda_{q}^{2}=0.4 \mathrm{GeV}^{2}$. In addition, we also employ the shorttailed platykurtic pion DA, which was more recently derived and discussed in $[8,40,41]$ using the same NLC QCD SR method but with a slightly larger value of the nonlocality parameter, notably, $\lambda_{q}^{2}=0.45 \mathrm{GeV}^{2}$. The platykurtic model DA is unimodal, like the asymptotic DA, but is much broader than this and has its tails $(x=0,1)$ suppressed like the classic bimodal BMS DA. It combines intrinsically the characteristic features of the $x$-distribution of the valence quark in the pion bound state being subject to DCSB as described via Dyson-Schwinger (DS) equations [42,43] — with correlations induced by NLCs in a nontrivial vacuum which give rise to a finite vacuum quark virtuality. The first effect, related to the mass dressing of the confined quark propagator, causes a broad (unimodal) downward concave shape of the DA with enhanced tails, while the second effect tends to suppress the endpoint regions and create two separated peaks. The reasons why the endpoint-regions of the pion DA should be suppressed, has been discussed in detail long ago, see e.g., [44,45], and more recently in [8,40] having recourse to the synchronization properties of nonlinear oscillators. In our estimates, shown in graphical form farther below, the BMS model DA is used to obtain the central TFF prediction, while the validity range of the BMS DAs [30] serves as a measure to estimate the involved intrinsic errors. Note that the "platykurtic" prediction for the pion-photon TFF almost coincides with that derived with the BMS model DA $[40,41]$.

\section{ASR approach}

In this section we briefly recall the main points of the ASR approach. This method relies upon the analysis of the vector-vector-axial current triangle graph amplitude

$$
T_{\alpha \mu \nu}(k, q)=\int d^{4} x d^{4} y e^{i(k \cdot x+q \cdot y)}\left\langle 0\left|T\left\{J_{\alpha 5}^{3}(0) J_{\mu}(x) J_{\nu}(y)\right\}\right| 0\right\rangle,
$$

where $k$ and $q$ are the momenta of the two photons, $J_{\alpha 5}^{3}$ is an isovector axial current, and $J_{\mu}$ and $J_{\nu}$ denote the electromagnetic currents. In what follows, we limit ourselves to the case when one of the photons is on-shell $\left(k^{2}=0\right)$.

It is convenient to write the tensor decomposition of this correlator in the form (see [28] for details)

$$
\begin{aligned}
T_{\alpha \mu \nu}(k, q)= & F_{1} \varepsilon_{\alpha \mu \nu \rho} k^{\rho}+F_{2} \varepsilon_{\alpha \mu \nu \rho} q^{\rho} \\
& +F_{3} k_{\nu} \varepsilon_{\alpha \mu \rho \sigma} k^{\rho} q^{\sigma}+F_{4} q_{\nu} \varepsilon_{\alpha \mu \rho \sigma} k^{\rho} q^{\sigma} \\
& +F_{5} k_{\mu} \varepsilon_{\alpha \nu \rho \sigma} k^{\rho} q^{\sigma}+F_{6} q_{\mu} \varepsilon_{\alpha \nu \rho \sigma} k^{\rho} q^{\sigma},
\end{aligned}
$$

where the coefficients $F_{j}=F_{j}\left(k^{2}, q^{2}, p^{2} ; m^{2}\right), p=k+q, j=1, \ldots, 6$ are the corresponding Lorentz invariant amplitudes, constrained by current conservation and Bose symmetry. Note that the latter includes the interchange $\mu \leftrightarrow \nu, k \leftrightarrow q$ in the tensor structures and $k^{2} \leftrightarrow q^{2}$ in the arguments of the scalar functions $F_{j}$. 
It was shown in [28] that the invariant amplitude $F_{3}$ satisfies the unsubtracted dispersion relation pertaining to the substraction of the axial-current divergence. (This seemingly controversial situation is due to the extra factor $q^{2}$ in the current divergence.) Using this unsubtracted dispersion relation, the dispersion representation of the axial anomaly for any virtual photon amounts to the ASR [28]

$$
\int_{0}^{\infty} A_{3}\left(s, Q^{2} ; m_{i}^{2}\right) d s=\frac{1}{2 \pi} N_{c} C,
$$

where $N_{c}=3$ is the number of colors, $C=\frac{1}{3 \sqrt{2}}$ is the charge factor, and $m_{i}$ are the quark masses. Here $Q^{2}=-q^{2}$ denotes the momentum transfer of the virtual photon, whereas $A_{3}$ is the imaginary part of the invariant amplitude $F_{3}$.

Although the spectral density $A_{3}\left(s, Q^{2} ; m^{2}\right)$ can in principle comprise both, perturbative as well as nonperturbative corrections, Eq. (9) is an exact expression and receives on its right-hand side neither perturbative corrections, by virtue of the AdlerBardeen theorem [46], nor nonperturbative contributions, the latter due to 't Hooft's principle [47]. ${ }^{1}$

To establish a relation of the above spectral density to the pion-photon TFF, we propose to saturate the three-point correlation function by means of resonances, notably, the pion state plus a continuum of all higher resonances. Then, assuming the validity of the global quark-hadron duality, we can express the contribution of all higher resonances as that part of the total integral in the ASR, i.e., the integral over the same spectral density $A_{3}\left(s, Q^{2} ; m^{2}\right)$, which starts from some lower limit $s_{0}$. Note that, in general, $s_{0}$ can depend on $Q^{2}$ and has the meaning of the pion duality interval. Taking into account that the pion resonances contribute via the pion-photon TFF, i.e., Eq. (3), and recalling that the original ASR is given in terms of Eq. (9), we finally obtain the following SR

$$
\pi f_{\pi} F_{\pi \gamma}\left(Q^{2}\right)=\frac{1}{2 \pi} N_{c} C-\int_{s_{0}}^{\infty} A_{3}\left(s, Q^{2} ; m_{i}^{2}\right) d s .
$$

Here, the pion decay constant $f_{\pi}$ is defined as the matrix element $\left\langle 0\left|J_{\alpha 5}(0)\right| M(p)\right\rangle=$ $i p_{\alpha} f_{\pi}$ and the pion-photon TFF $F_{\pi \gamma}\left(Q^{2}\right)$ is given by Eq. (3).

The contribution to the spectral density $A_{3}\left(s, Q^{2} ; m^{2}\right)$ for a given flavor $q$ is [28],

$$
A_{3}^{(q)}\left(s, Q^{2} ; m_{q}^{2}\right)=\frac{e_{q}^{2}}{2 \pi} \frac{1}{\left(Q^{2}+s\right)^{2}}\left(Q^{2} R+2 m_{q}^{2} \ln \frac{1+R}{1-R}\right),
$$

where $R\left(s, m_{q}^{2}\right)=\sqrt{1-\frac{4 m_{q}^{2}}{s}} m_{q}$ is the quark mass for flavor $q$. Neglecting the quark mass, we obtain from (10) and (11) the following expression for the pion-photon TFF,

$$
F_{\pi \gamma}\left(Q^{2}\right)=\frac{1}{2 \sqrt{2} \pi^{2} f_{\pi}} \frac{s_{0}\left(Q^{2}\right)}{s_{0}\left(Q^{2}\right)+Q^{2}},
$$

\footnotetext{
${ }^{1}$ It is worth mentioning that the first-order correction $\propto \alpha_{s}$ to the integrand itself is zero - at least in the massless limit, see [48-50].
} 
where $f_{\pi}=0.134 \mathrm{MeV}$ is the pion decay constant.

Note that the ASR relation is valid for any value of $Q^{2}$ (given that the total integral does not depend on it). But, in addition, also relation (12) is valid for all $Q^{2}$ values, hence defining an exact property of the TFF. On the most basic level, one obviously recovers for $Q^{2}=0$ the chiral limit $\frac{1}{2 \sqrt{2} \pi^{2} f_{\pi}} F_{\pi \gamma}\left(Q^{2}=0\right)=1$. Moreover, the continuation of (12) from the spacelike region to the timelike domain can also be performed [29]. This procedure does not violate the exactness of the ASR, viz., Eq. (9), provided one assumes that $s_{0}$ has the most general form which allows for a dependence on the momentum transfer [51]. Let us assume that, though $s_{0}$ resembles the so-called continuum threshold parameter in QCD sum rules, it may vary with $Q^{2}$ like in the KOT approach. In addition, it may also have different values in the twoand three-point correlators. Be that as it may, as long as we do not fix the form of $s_{0}\left(Q^{2}\right)$, the ASR result for the pion-photon TFF given by Eq. (12) remains an exact relation. One has to fix the form of $s_{0}\left(Q^{2}\right)$ only if one is interested in the numerical value of this quantity. This may eventually entail some inaccuracy, like in the KOT approach.

On the pretext that $s_{0}$ is a continuum threshold parameter, we may suppose that in first approximation $s_{0}$ is a constant. With this assumption, $s_{0}$ can be determined from Eq. (12) in the limit $Q^{2} \rightarrow \infty$ of the spacelike ASR (see [22]). As in the asymptotic limit, the validity of the factorization theorem should be completely restored, so that one easily obtains $s_{0}=4 \pi^{2} f_{\pi}^{2} \simeq 0.67 \mathrm{GeV}^{2}$. This expression coincides with the one found earlier from a two-point correlator analysis in [52] and is also close to the numerical value estimated using two-point sum rules [53]. In this way, we find that the Brodsky-Lepage interpolation formula [3] (which corresponds to the one-loop approximation of the ASR) holds in the timelike region as well. ${ }^{2}$ It is also worth mentioning that an expression similar to Eq. (12) was derived in [55] by employing an extension of VMD ideas to include higher resonances in combination with the correct large- $Q^{2}$ behavior of the TFF.

In this paper we want to scrutinize the assumption that $s_{0}$ can be supposed to be close to a constant. To this end, we will assume that $s_{0}$ is some arbitrary (but smooth) function of $Q^{2}$ and then compare the ASR relation for the TFF, cf. (12), with the predictions obtained within the BMPS LCSR-based analysis in [15, 16, 18].

\section{Interplay between lightcone and anomaly sum rules}

In this section, we will work out the connection between the two sum-rule approaches considered above. Our comparative analysis will be carried out in three steps:

\footnotetext{
${ }^{2}$ The similarity between the Brodsky-Lepage interpolation formula in the spacelike region and the vector-dominance model in the timelike region is well known, see, e.g., [54].
} 


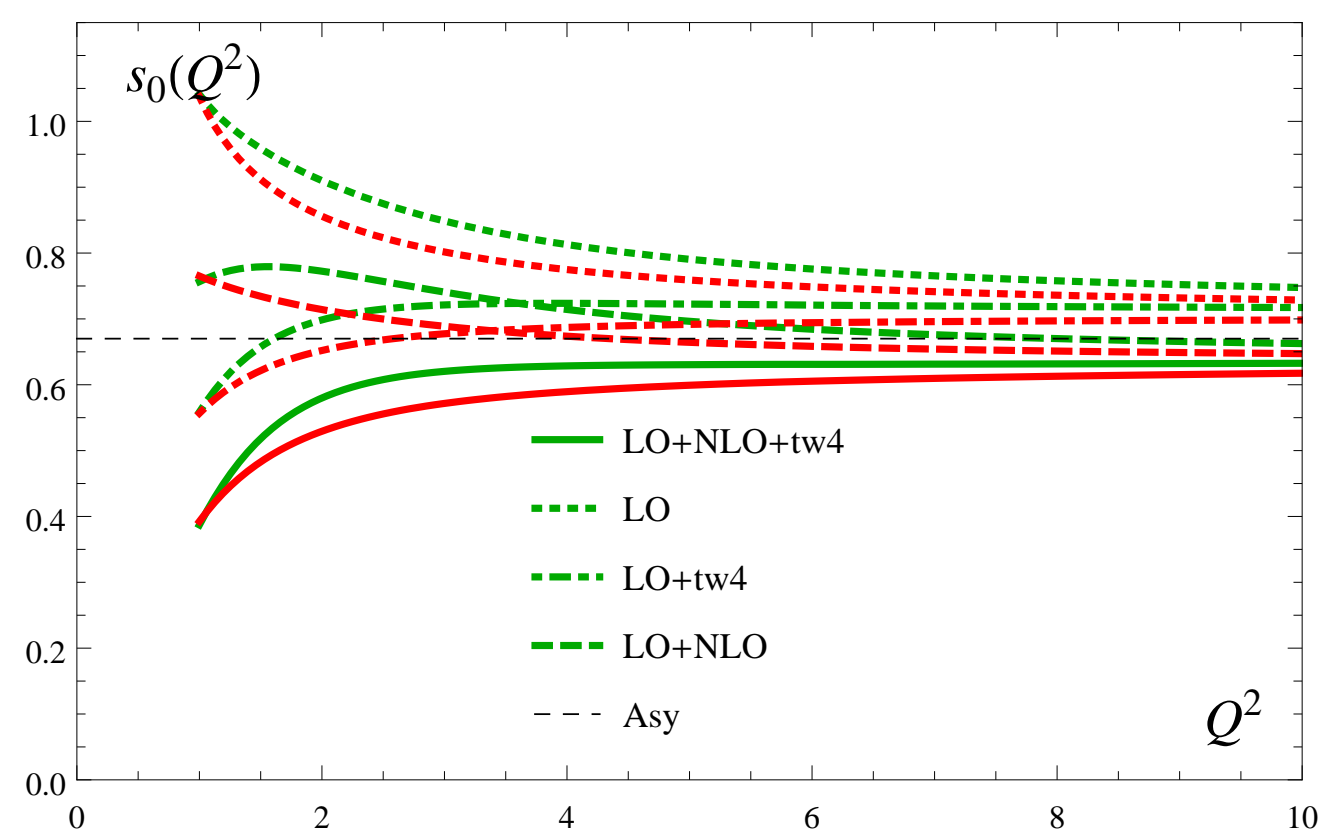

Figure 1: (Color online). Anomaly SR threshold parameter $s_{0}\left(Q^{2}\right)$ extracted from the comparison of the pion-photon TFF obtained from the anomaly SR and from LCSRs at four levels of accuracy: (i) dotted lines - LO result; (ii) dashed lines - LO+NLO; (iii) dashed-dotted lines - LO with twist four contribution included; (iv) thick solid line - LO+NLO+twist- 4 . The horizontal dashed line represents the threshold value $s_{0}=4 \pi^{2} f_{\pi}^{2} \simeq 0.67 \mathrm{GeV}^{2}$. The green lines show the results for the BMS pion DA model [30], whereas the red lines display the analogous results for the shorttailed platykurtic pion DA [41]. Note that from top to bottom, all red curves appear always below the corresponding green ones.

1. Presuming that the threshold $s_{0}\left(Q^{2}\right)$ is some definite (but unknown) function of the momentum, we estimate its value by comparing (12) with (4) within the validity range of the LCSR approach $\left(Q^{2} \gtrsim 1 \mathrm{GeV}^{2}\right)$. The outcome of this comparison is shown in Fig. 1. The various lines correspond to different contributions, taken into account step by step by means of LCSRs. Their meaning is explained from top to bottom. Line one (dotted) shows the LO result, while the next lower one (dashed) illustrates an analogous result when also the NLO term has been included in the TFF. The third line (dashed-dotted) represents the prediction which includes only the LO term and the twist-four contribution, with the NLO term being excluded. Finally, the lowest curve (thick solid) displays the total result which comprises the NLO radiative contribution as well as the twist-four term. In addition, also the limiting case $s_{0}=0.67 \mathrm{GeV}^{2}$, obtained from ASRs under the assumption that $s_{0}$ is constant, is shown in the form of a horizontal dashed line. The analysis was carried out using two different models for the 


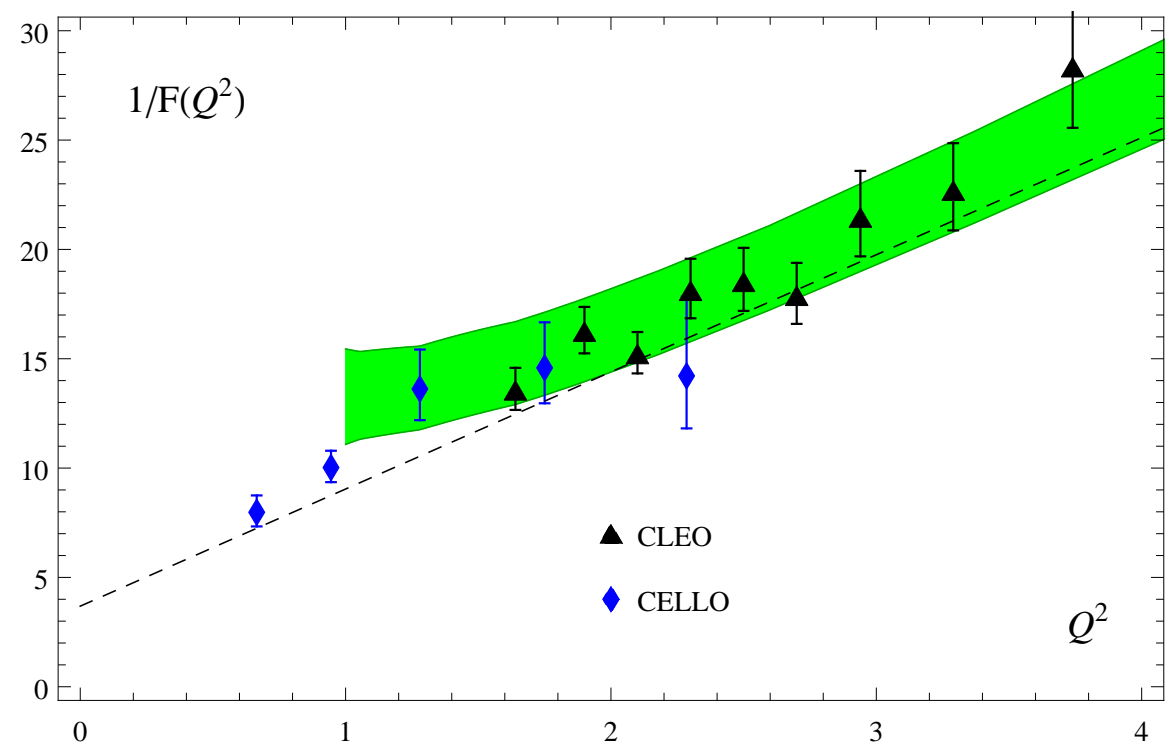

Figure 2: (Color online). Inverse pion-photon TFF obtained from two different sumrule approaches: LCSR (broad green band) and linear behavior from anomaly SR (dashed line). The experimental data are taken from CELLO [54] — blue diamonds and CLEO [56] — black triangles.

pion DA: the bimodal BMS DA [30] (green lines) and the shorttailed platykurtic DA [41] (red lines - always below the green ones).

One observes from this figure that below $2-3 \mathrm{GeV}^{2}$ the various curves start to deviate significantly from each other calling for more detailed considerations to be addressed in the next item. On the other hand, it is worth noting that above $Q^{2} \gtrsim 3 \mathrm{GeV}^{2}, s_{0}\left(Q^{2}\right)$ starts to scale with $Q^{2}$ and has a constant magnitude that depends on the actual approximation of the LCSR. Let us note that the total result for both, the BMS pion DA and the platykurtic DA, leads to a $s_{0}$ value rather far from the constant $0.67 \mathrm{GeV}^{2}$ used in the KOT approach even in the intermediate region $Q^{2}<10 \mathrm{GeV}^{2}$. On the other hand, in the NLO approximation of the LCSR ( $\mathrm{LO}+\mathrm{NLO}$ without twist 4 contribution), expressions (12) and (4) numerically coincide, thus confirming the assumption that $s_{0}$ is close to a constant. The explanation of this coincidence will be discussed shortly.

2. Let us concentrate on the low-momentum region below $3 \mathrm{GeV}^{2}$. Here, the role of higher-order corrections to the LCSR, both perturbative and nonperturbative, becomes significant, whereas the ASR result (according to the KOT assumption) does not change, being $Q^{2}$ independent. This becomes evident from the comparison of the LO TFF prediction with the one which includes the NLO correction, employing in both cases the BMS DA within the BMPS framework. As one can see from Fig. 1, the LO approximation to the LCSR leads to an unreliable depen- 
dence on $Q^{2}$, even if the twist-four contribution is incorporated. It is only when the NLO term is taken into account that the result stabilizes, leading to a $s_{0}$ close to a constant. This behavior reflects the fact that at such virtualities perturbative, i.e., radiative, corrections to the LCSR are vital. It is remarkable that using instead the shorttailed platykurtic DA, one finds that $s_{0}$ almost coincides with the result derived in the KOT ASR in the entire range of $Q^{2}$. In contrast, incorporating only the twist-four contribution renders also this result worse, remaining bad even after the inclusion of the NLO term, as already mentioned. In both cases, the value of $s_{0}$ decreases appreciably and, as a result, stability against the $Q^{2}$ variation gets worse as well.

One should note, however, that effects stemming from large-distance dynamics are accounted for by the anomaly relation itself "as a whole", implying that to compare its prediction with that obtained with LCSRs, one should include in the latter the sum over the whole infinite series of the nonperturbative corrections [57]. The absence of a nonperturbative correction to the ASR according to t'Hooft's principle may indicate that a strong cancellation of nonperturbative corrections takes place. Indeed, estimations in [16] show that the next term in the nonperturbative expansion (the twist-six contribution) has the opposite sign relative to the twist four term and should therefore grossly cancel it's contribution. Moreover, one may provide additional arguments that a significant cancellation of the nonperturbative corrections may indeed take place. In fact, if one expands the exact ASR result (12) for the pion TFF in a series in terms of the ratio $s_{0} / Q^{2}$, one obtains an expression which could be regarded as an infinite-series expansion of the nonperturbative corrections. One can easily see that, for the physically reliable case of $s_{0}$ being close to a constant (or at least a not strongly oscillating function of $Q^{2}$ ), the series should be an alternating one.

This clearly means that at momentum values around $Q^{2}=1-2 \mathrm{GeV}^{2}$, a strong cancelation of the perturbative corrections should take place. Thus, we may come to the conclusion that the ASR result should be compared with the prediction derived from the LCSR only when the NLO term is included while the twistfour contribution is excluded. For this case one may further conclude from Fig. 1 that the comparison with the BMPS result (using the BMS DA) supports the assumption that the pion duality interval $s_{0}\left(Q^{2}\right)$ in the KOT approach is close to a constant (second line from the top in this figure), implying that the approximation $s_{0}=0.67 \mathrm{GeV}^{2}$ is reasonable within a 10 percent accuracy. Note that employing the shorttailed platykurtic pion DA, this agreement becomes even better.

3. For a more accurate analysis of the TFF, it is convenient to analyze the behavior of the pion-photon TFF at $q^{2}=-Q^{2} \rightarrow 0$ in terms of the ratio $R \equiv$ $F_{\pi \gamma}\left(q^{2}\right) / F_{\pi \gamma}(0)$. The dimensionless slope and curvature parameters at $q^{2}=0$, are defined by $a=m_{\pi}^{2} \partial R /\left.\partial q^{2}\right|_{q^{2}=0}$ and $b=\frac{1}{2} m_{\pi}^{4} \partial^{2} R /\left.\partial\left(q^{2}\right)^{2}\right|_{q^{2}=0}$, respectively, and 
have for the pion the following values: $a_{\pi}=m_{\pi}^{2} / s_{0}=0.027$ and $b_{\pi}=m_{\pi}^{4} / s_{0}^{2}=$ $0.73 \times 10^{-3}$. The slope $a_{\pi}$ was recently determined in [58] by means of Padé approximants as fitting functions in the analysis of the spacelike experimental data of the pion-photon TFF and was found to be $a_{\pi} \simeq 0.0324$. A more recent dispersive analysis in [59] extracted at vanishing momentum transfer the value $a_{\pi} \simeq 0.0307$. Both results for the slope are (within the range of the estimated errors omitted here) compatible with each other and also in good agreement with the ASR result within the $10 \%$ margin of accuracy at the lower limit discussed before. From (12) one can easily see that the slope of the TFF at $q^{2}=0$ depends only on the value $s_{0}$ due to $d R / d q^{2}=1 / s_{0}$. Thus, we can conclude that, though $s_{0}\left(q^{2}\right)$ can be treated as constant to the level of 10 percent precision, a more accurate treatment would be to start at the value $s_{0}=0.61 \mathrm{GeV}^{2}$ for $q^{2}=0$ and approach the asymptotic value $s_{0}=0.67 \mathrm{GeV}^{2}$ at the scale $-q^{2}=Q^{2}=1-2 \mathrm{GeV}^{2}$. It is worth noting that the value $s_{0}=0.61 \mathrm{GeV}^{2}$ at $q^{2}=0$ coincides with the $\rho$-meson mass squared. The slope $a_{\pi}$ can be related to the pion radius by the trivial relation $\left(\left\langle r_{\pi}\right\rangle\right)^{2}=6 a_{\pi} / m_{\pi}^{2}$ (see [60] for a discussion). In this way we find the pion radius for $s_{0}=0.61 \mathrm{GeV}^{2}$ to be $0.62 \mathrm{fm}$, while for the asymptotic value $s_{0}=0.67 \mathrm{GeV}^{2}$ the result is slightly smaller and about $0.59 \mathrm{fm}$. Both values are in accord with the pion radius derived from considerations based on the vector-meson dominance, whereas the pion radius extracted from the DS-based approach [42] turns out to be somewhat larger, $0.68 \mathrm{fm}$.

As regards the curvature parameter $b_{\pi}$ at $q^{2}=0$, it was computed in the works mentioned above and found to be $b_{\pi} \approx 0.0011$ - see $[58,59]$ for the corresponding computational details. Comparing this value with the ASR result shows that agreement can be achieved at $s_{0}=0.61 \mathrm{GeV}^{2}$ (with $q^{2}=0$ ), allowing for the $s_{0}$ derivative to be negative, $d s_{0} / d q^{2}=-d s_{0} / d Q^{2}=-0.25$. In summary, it is reasonable to expect that $s_{0}$ is $0.61 \mathrm{GeV}^{2}$ at the zero point and grows with $Q^{2}$ up to the asymptotic value $s_{0}=0.67 \mathrm{GeV}^{2}$ at scales on the order of $1-2 \mathrm{GeV}^{2}$.

4. It was noted in Ref. [29] that analytic continuation of the ASR approach to the timelike region leads to a pole in the TFF at $q^{2}=s_{0}$, which is numerically close to the $\rho$ meson mass squared, i.e., $m_{\rho}^{2} \simeq 0.59 \mathrm{GeV}^{2}$. This pole can be traced back to the analytic continuation of the ASR into the timelike domain and arises because in the spacelike region the TFF is proportional to $s_{0} /\left(s_{0}-q^{2}\right)$. As a result, the inverse TFF $1 / F\left(Q^{2}\right)$ should show a quasi linear behavior at moderately large $Q^{2}$. Furthermore, it should also agree with the well-known limit of the TFF for two real photons (i.e, the two-photon pion decay width). Because the LCSRs are not reliably applicable at very low momenta below, say, below $1 \mathrm{GeV}^{2}$, it looks promising to compare the KOT results at low $Q^{2}=-q^{2}>0$ in the spacelike region with the experimental data and to consider a possible (linear) interpolation of the BMS prediction to the well-known limit of the TFF for two real photons (i.e., the two-photon decay width, which clearly does not depend on 
the $s_{0}$ value). Therefore, we show in Fig. 2 the predictions for the inverse TFF $1 / F\left(Q^{2}\right)$ obtained with the KOT approach with a constant $s_{0}$ value (solid line) and also with the BMPS method, contrasting them with the experimental data at low $Q^{2}$. The BMPS predictions are shown by means of a shaded (green) area which indicates its accuracy range. The slight excess of the BMS results can be interpreted as a reflection of the small $(\sim 10 \%)$ variation of $s_{0}\left(Q^{2}\right)$ at small $Q^{2}$ and uncertainties originating from the LCSRs themselves at small $Q^{2}$ values.

We see from Fig. 2 that the ASR result (solid line) is in good agreement with the experimental data and exactly corresponds to the two-photon decay width at $Q^{2}=0$. On the other hand, the LCSR prediction also conforms with experiment - starting from about $2 \mathrm{GeV}^{2}$ - and allows for a linear interpolation of $1 / F\left(Q^{2}\right)$ to the twophoton decay width at $Q^{2}=0$. However, the LCSR result for the inverse TFF $1 / F\left(Q^{2}\right)$ starts deviating from the linear interpolation formula at lower momenta in the region 1-1.5 $\mathrm{GeV}^{2}$ where the LCSRs are supposed to be still reliable. Indeed, a smooth continuous interpolation (including the derivative) to the limit of the two-photon decay width is not showing a linear behavior. This indicates that $s_{0}$ may deviate from a constant at small $Q^{2}<1.5 \mathrm{GeV}^{2}$ at the level of $10-20 \%$. This deviation may be the result of uncertainties of the LCSR method at small momentum scales currently under investigation [61], see also [16], where the interplay between the main NNLO contribution ( $\beta_{0}$-part) and the twist-six term is discussed. If this deviation would be confirmed by further analysis, it would be very interesting to estimate its analytic form, especially bearing in mind that, after the analytic continuation to the timelike region (see Ref. [29]), the deviation from linearity could be related to the value of the widths of vector mesons. Unfortunately the current knowledge of the intrinsic uncertainties of the LCSR predictions in the low $Q^{2}$ domain is quite limited to allow a precise estimation of this deviation. We hope that the dedicated low $Q^{2}$ analysis of the LCSRs in [61] will provide more clues.

\section{Conclusions}

In this work we performed a comparative analysis of the pion-photon TFF computed with two different types of sum rules: LCSRs and ASRs. The first method interpolates correctly the behavior of the electromagnetic pion-photon transition form factor for a highly virtual and a real photon from the ultraviolet limit of QCD down to typical hadronic scales of about $1-2 \mathrm{GeV}$, close to the dipole formula. The second method is based on the chiral anomaly and is exact even when both photons have vanishing virtualities. The objective was to match the key parameters of these approaches in the low- $Q^{2}$ domain thus, in some sense, "unifying" their predictions. To this end, we used within the BMPS LCSR approach two different types of pion DAs. The classic bimodal BMS DAs [30] and the unimodal shorttailed platykurtic DA [8,40]. The common key element of both DAs is the strong suppression of the kinematic endpoint 
regions $x=0,1$. This behavior distinguishes them from many other model DAs, ranging from the bimodal endpoint-concentrated Chernyak-Zhitnitsky [62] DA to the recently proposed DSE-based DAs which have broad unimodal profiles with strongly enhanced tails. The main results of our analysis have been presented graphically in Fig. 1 and Fig. 2. From the first figure we observe that the pion-photon TFF, computed with the platykurtic DA within the LCSR framework, strongly resembles the analogous result computed with the ASRs. However, this mutual consistency is best only if the twist-four contribution in the former calculation is excluded, arguing that the ASR prediction inherently embodies not only the first next-to-leading-order twist term, but a whole series of such contributions. Moreover, from this agreement one can infer that the threshold parameter $s_{0}$ in the ASR approach is a constant with a matching value $s_{0}=0.67 \mathrm{GeV}^{2}$ at the accuracy level of $10 \%$. On the other hand, inspection of Fig. 2 reveals that strong cancelations of the radiative corrections are needed in order to produce the linear behavior of the displayed ASR result (dashed line). But, from the other side, if the observed deviation is real it might indicate a dependence of $s_{0}$ on the large photon virtuality $Q^{2}$. From our combined analysis we found that the value $s_{0}$ can vary between the value $0.61 \mathrm{GeV}^{2}$ at $Q^{2}=0$ and the asymptotic value $0.67 \mathrm{GeV}^{2}$ at the scale $1-2 \mathrm{GeV}^{2}$. We also found by employing the ASR approach that the pion radius can be estimated to be in the interval $0.59-0.62 \mathrm{fm}$.

\section{Acknowledgments}

We thank Y. N. Klopot and S. V. Mikhailov for useful discussions and comments. This work was partially supported by the Heisenberg-Landau Program (Grant 2015), the Russian Foundation for Basic Research under Grants No. 14-01-00647 and No. 15-52-04023, the JINR-BelRFFR grant F14D-007, the Major State Basic Research Development Program in China (No. 2015CB856903), and the National Natural Science Foundation of China (Grant Nos. 11575254 and 11175215).

\section{References}

[1] B. Aubert et al., Phys. Rev. D80, 052002 (2009).

[2] G. P. Lepage and S. J. Brodsky, Phys. Rev. D22, 2157 (1980).

[3] S. J. Brodsky and G. P. Lepage, Phys. Rev. D24, 1808 (1981).

[4] S. Uehara et al., Phys. Rev. D86, 092007 (2012).

[5] S. V. Mikhailov and N. G. Stefanis, Nucl. Phys. B821, 291 (2009).

[6] A. V. Radyushkin, Phys. Rev. D80, 094009 (2009). 
[7] M. V. Polyakov, JETP Lett. 90, 228 (2009).

[8] N. G. Stefanis and A. V. Pimikov, Nucl. Phys. A945, 248 (2016).

[9] A. V. Efremov and A. V. Radyushkin, Phys. Lett. B94, 245 (1980).

[10] I. I. Balitsky, V. M. Braun, and A. V. Kolesnichenko, Nucl. Phys. B312, 509 (1989).

[11] A. Khodjamirian, Eur. Phys. J. C6, 477 (1999).

[12] A. P. Bakulev, S. V. Mikhailov, and N. G. Stefanis, Phys. Rev. D67, 074012 (2003).

[13] A. P. Bakulev, S. V. Mikhailov, and N. G. Stefanis, Phys. Lett. B578, 91 (2004).

[14] A. P. Bakulev, S. V. Mikhailov, and N. G. Stefanis, Phys. Rev. D73, 056002 (2006).

[15] S. V. Mikhailov, A. V. Pimikov, and N. G. Stefanis, Phys. Rev. D82, 054020 $(2010)$.

[16] A. V. Bakulev, S. V. Mikhailov, A. V. Pimikov, and N. G. Stefanis, Phys. Rev. D84, 034014 (2011).

[17] A. P. Bakulev, S. V. Mikhailov, A. V. Pimikov, and N. G. Stefanis, Phys. Rev. D86, 031501 (2012).

[18] N. G. Stefanis, A. V. Bakulev, S. V. Mikhailov, and A. V. Pimikov, Phys. Rev. D87, 094025 (2013).

[19] A. Khodjamirian, Int. J. Mod. Phys. A25, 513 (2010).

[20] S. S. Agaev, V. M. Braun, N. Offen, and F. A. Porkert, Phys. Rev. D83, 054020 (2011).

[21] S. S. Agaev, V. M. Braun, N. Offen, and F. A. Porkert, Phys. Rev. D86, 077504 (2012).

[22] Y. N. Klopot, A. G. Oganesian, and O. V. Teryaev, Phys. Lett. B695, 130 (2011).

[23] Y. N. Klopot, A. G. Oganesian, and O. V. Teryaev, Phys. Rev. D84, 051901 (2011).

[24] Y. N. Klopot, A. G. Oganesian, and O. V. Teryaev, JETP Lett. 94, 729 (2011).

[25] Y. N. Klopot, A. G. Oganesian, and O. V. Teryaev, Phys. Rev. D87, 036013 (2013). 
[26] A. D. Dolgov and V. I. Zakharov, Nucl. Phys. B27, 525 (1971).

[27] J. Horejsi, Phys. Rev. D32, 1029 (1985).

[28] J. Horejsi and O. V. Teryaev, Z. Phys. C65, 691 (1995).

[29] Y. N. Klopot, A. G. Oganesian, and O. V. Teryaev, JETP Lett. 99, 679 (2014).

[30] A. P. Bakulev, S. V. Mikhailov, and N. G. Stefanis, Phys. Lett. B508, 279 (2001); ibid. B590 (2004), 309 (Erratum).

[31] A. Schmedding and O. Yakovlev, Phys. Rev. D62, 116002 (2000).

[32] S. V. Mikhailov and A. V. Radyushkin, JETP Lett. 43, 712 (1986).

[33] S. V. Mikhailov and A. V. Radyushkin, Nucl. Phys. B273, 297 (1986).

[34] S. V. Mikhailov and A. V. Radyushkin, Sov. J. Nucl. Phys. 49, 494 (1989).

[35] S. V. Mikhailov and A. V. Radyushkin, Sov. J. Nucl. Phys. 52, 697 (1990).

[36] S. V. Mikhailov, Phys. Atom. Nucl. 56, 650 (1993).

[37] A. P. Bakulev and A. V. Radyushkin, Phys. Lett. B271, 223 (1991).

[38] S. V. Mikhailov and A. V. Radyushkin, Phys. Rev. D45, 1754 (1992).

[39] B. Melić, D. Müller, and K. Passek-Kumerički, Phys. Rev. D68, 014013 (2003).

[40] N. G. Stefanis, Phys. Lett. B738, 483 (2014).

[41] N. G. Stefanis, S. V. Mikhailov, and A. V. Pimikov, Few Body Syst. 56, 295 (2015).

[42] L. Chang et al., Phys. Rev. Lett. 110, 132001 (2013).

[43] F. Gao et al., Phys. Rev. D90, 014011 (2014).

[44] N. G. Stefanis, W. Schroers, and H.-C. Kim, Phys. Lett. B449, 299 (1999).

[45] N. G. Stefanis, W. Schroers, and H.-C. Kim, Eur. Phys. J. C18, 137 (2000).

[46] S. L. Adler, Phys. Rev. 177, 2426 (1969).

[47] G. 't Hooft et al., NATO Sci.Ser.B 59, pp.1 (1980).

[48] F. Jegerlehner and O. V. Tarasov, Phys. Lett. B639, 299 (2006).

[49] R. S. Pasechnik and O. V. Teryaev, Phys. Rev. D73, 034017 (2006). 
[50] A. L. Kataev, JHEP 1402, 092 (2014).

[51] D. Melikhov and B. Stech, Phys. Lett. B718, 488 (2012).

[52] A. V. Radyushkin, Acta Phys. Polon. B26, 2067 (1995).

[53] M. A. Shifman, A. Vainshtein, and V. I. Zakharov, Nucl. Phys. B147, 448 (1979).

[54] H. J. Behrend et al., Z. Phys. C49, 401 (1991).

[55] P. Masjuan, E. Ruiz Arriola, and W. Broniowski, Phys. Rev. D87, 014005 (2013).

[56] J. Gronberg et al., Phys. Rev. D57, 33 (1998).

[57] O. V. Teryaev, Nucl. Phys. Proc. Suppl. 245, 195 (2013).

[58] P. Masjuan, Phys. Rev. D86, 094021 (2012).

[59] M. Hoferichter et al., Eur. Phys. J. C74, 3180 (2014).

[60] A. M. Bernstein, in 8th International Workshop on Chiral Dynamics (CD 2015) Pisa, Italy, June 29-July 3, 2015 (PUBLISHER, ADDRESS, 2015).

[61] S. V. Mikhailov, A. V. Pimikov, and N. G. Stefanis, Bochum preprint RUB-TPII04/2015, work in progress.

[62] V. L. Chernyak and A. R. Zhitnitsky, Phys. Rept. 112, 173 (1984). 